OPEN ACCESS

Edited by:

Rosaria Maria Pipitone,

University of Palermo, Italy

Reviewed by:

Xiongwen LV,

Anhui Medical University, China

Jianping Cao,

National Institute of Parasitic Diseases,

China

${ }^{*}$ Correspondence:

Yinong Duan

yinongduan@aliyun.com

Lian Duan

duanlian@ntu.edu.cn

${ }^{\dagger}$ These authors have contributed equally to this work

Specialty section:

This article was submitted to Molecular and Cellular Pathology, a section of the journal

Frontiers in Cell and Developmental

Biology

Received: 27 August 2021

Accepted: 22 October 2021

Published: 08 November 2021

Citation:

Li J, Zhang J, Zhang B, Chen L, Chen G, Zhu D, Chen J, Duan L and

Duan Y (2021) rSjP40 Inhibited the Activity of Collagen Type I Promoter via

Ets-1 in HSCs.

Front. Cell Dev. Biol. 9:765616.

doi: $10.3389 /$ fcell.2021.765616

\section{rSjP40 Inhibited the Activity of Collagen Type I Promoter via Ets-1 in HSCs}

\author{
Jing $\mathrm{Li}^{1,2+}$, Jiali Zhang ${ }^{1,3+}$, Bei Zhang ${ }^{1 \dagger}$, Liuting Chen ${ }^{1}$, Guo Chen ${ }^{1}$, Dandan Zhu ${ }^{1}$, \\ Jinling Chen ${ }^{1}$, Lian Duan ${ }^{4 *}$ and Yinong Duan ${ }^{1 *}$
}

${ }^{1}$ Department of Pathogen Biology, School of Medicine, Nantong University, Nantong, China, ${ }^{2}$ Cancer Research Center Nantong, Nantong Tumor Hospital \& Tumor Hospital Affiliated to Nantong University, Nantong, China, ${ }^{3}$ Department of Laboratory, Xishan People's Hospital of Wuxi City, Wuxi, China, ${ }^{4}$ Department of Medical Informatics, School of Medicine, Nantong University, Nantong, China

Liver fibrosis is a severe disease characterized by excessive deposition of extracellular matrix (ECM) components in the liver. Activated hepatic stellate cells (HSCs) are a major source of ECM and a key regulator of liver fibrosis. Collagen type I alpha I (COL1A1) is one of the main components of ECM and is a major component in fibrotic tissues. Previously, we demonstrated that soluble egg antigen from Schistosoma japonicum could inhibit the expression of COL1A1 in activated HSCs. In addition, studies have found that Ets protooncogene 1 (Ets-1) suppresses the production of ECM by down-regulating matrix related genes such as COL1A1 induced by transforming growth factor $\beta$, and ultimately inhibits liver fibrosis. In this study, the major aim was to investigate the effect and mechanism of Ets-1 on inhibiting COL1A1 gene promoter activity in HSCs by recombinant Schistosoma japonicum protein P40 (rSjP40). We observed the rSjP40 inhibited the expression of COL $1 A 1$ by inhibiting the activity of the COL1A1 promoter, and the core region of rSjP40 acting on COL1A1 promoter was located at $-1,722 /-1,592$. In addition, we also demonstrated that rSjP40 could promote the expression of Ets-1, and Ets-1 has a negative regulation effect on the COL1A1 promoter in human LX-2 cells. These data suggest that $\mathrm{rSjP} 40$ might inhibit the activity of COL $1 A 1$ promoter and inhibit the activation of HSCs by increasing the expression of transcription factor Ets-1, which will provide a new experimental basis for the prevention and treatment of liver fibrosis.

Keywords: schistosoma japonicum protein P40, hepatic stellate cells, collagen type I alpha I, liver fibrosis, ETS-1

\section{INTRODUCTION}

Liver fibrosis is a common pathological change of chronic liver disease, which usually occurs after chronic liver injury. It is known that the main processes of liver fibrosis are the activation of hepatic stellate cells (HSCs) and subsequently the excessive deposition of extracellular matrix (ECM) components (Udomsinprasert and Jittikoon, 2019). After the inflammation continues to develop in the liver, HSCs are activated from a resting state to an activated myofibroblast phenotype (Yin et al., 2013; Fabregat and Caballero-Diaz, 2018), which then leads to an imbalance of the formation and degradation of ECM proteins (Tang, 2015). Collagen is the main component of extracellular matrix and the dominant component in fibrotic tissue (Yang et al., 2017). The major types of collagen in liver fibrosis include type I, III and IV. The massive deposition of collagen type I alpha I (COL1A1) 
and collagen III in the interstitium is a sign of advanced hepatic fibrosis (Chatterjee et al., 2005). In the early stage of fibrosis, collagen III is slightly increased, while COL1A1 is highly increased, and it is still the main type of collagen later on (Eckes et al., 2000). Therefore, the expression level of COL1A1 can reflect the progression of liver fibrosis, inhibiting its expression will be one of the effective measures to reduce liver fibrosis.

Schistosomiasis is a common parasitic disease induced by schistosomes. The main pathogenesis of schistosomiasis is the occurrence of egg granuloma in the liver and intestinal wall, which can lead to severe enterohepatic fibrosis (Duan et al., 2014). Soluble egg antigen (SEA) contains a variety of antigen components and Schistosoma japonicum protein $\mathrm{P} 40(\mathrm{SjP} 40)$ is one of the main components in the SEA, which belongs to the heat shock protein family of schistosomiasis (Zhu et al., 2018). Previous studies in our lab have shown that SEA from Schistosoma japonicum could inhibit the expression of COL1A1 in activated HSCs (Duan et al., 2014). We also confirmed that the recombinant $\mathrm{SjP} 40$ protein $(\mathrm{rSjP} 40)$ could inhibit the activation and proliferation of HSCs (Sun et al., 2015). However, the contribution and the mechanism of SjP40 in inhibiting the expression of COL1A1 in activated HSCs have not been demonstrated.

Ets proto-oncogene 1 (Ets-1), a member of the Ets transcription factor family, has been reported to be involved in fibrosis progression by regulating fibroblast activation and proliferation, and regulating the synthesis and degradation of ECM. Studies have found that upregulation of Ets-1 expression enhances transforming growth factor $\beta$ (TGF- $\beta$ )-induced hepatocyte apoptosis and accelerates liver inflammation and fibrosis in nonalcoholic steatohepatitis (NASH) mice (Liu et al., 2019). However, it has been reported that Ets-1 suppresses the production of ECM by down-regulating matrix related genes such as COL1A1 induced by TGF- $\beta$, and ultimately inhibit liver fibrosis (Ozaki et al., 2002; Ozaki et al., 2003; Liu et al., 2016). However, the role of Ets-1 in the inhibition of COL1A1 expression by $\mathrm{rSjP} 40$ remains unclear.

In this study, we observed that whether the transcription factor Ets-1 was participated in the inhibition of COL1A1 in human HSC cell lines LX-2 cells treated with rSjP40.

\section{MATERIALS AND METHODS}

\section{Reagents}

We obtained rSjP40 protein as previously described (Chen et al., 2016b). Mouse mAbs against COL1A1 (Abcam, United Kingdom), rabbit mAbs against GAPDH (Goodhere, China) or Ets-1 (Proteintech, United Kingdom), horseradish peroxidase (HRP)-conjugated anti-mouse IgG (Santa Cruz, United States) and HRP-conjugated anti-rabbit IgG (Biosharp, China) were purchased from the indicated companies. Plasmids containing COL1A1 promoter sequences constructed as previously described (Chen et al., 2019a) were preserved in our lab.

\section{Cell Culture and Treatment}

LX-2 cell, a human HSC line, were obtained from Nantong Third People's Hospital and cultured in Dulbecco's Modified Eagle's Medium (DMEM, Gibco, United States) supplemented with 10\% FBS (Excel, China). Cells were maintained in a humidified incubator containing $5 \% \mathrm{CO}_{2}$ at $37^{\circ} \mathrm{C}$, inoculated into 12 or 24 well culture-plates and then treated with $\mathrm{rSjP} 40$.

\section{Western Blot}

Cell lysates were prepared on ice for extracting proteins using RIPA buffer containing protease inhibitor $(1 \mathrm{mM})$ and phosphatase inhibitors $(1 \mathrm{mM})$. An equal amount of each lysate $(50 \mu \mathrm{g})$ was separated by $10 \%$ sodium dodecyl sulfatepolyacrylamide gel electrophoresis. After the proteins were transferred onto polyvinylidene difluoride (PVDF, Millipore, United States) membranes, the membranes were blocked in TBST containing 5\% nonfat milk for $1 \mathrm{~h}$ and then incubated with the indicated primary antibodies diluted with $5 \%$ nonfat milk overnight at $4 \mathrm{C}$. The primary antibodies used for the western blot were as follows: mouse antibody against COL1A1 (Abcam, United Kingdom) (1:200 dilution), and rabbit antibody against Ets-1 (Proteintech, United Kingdom) (1:500 dilution) or GAPDH (Goodhere, China) (1:1,000 dilution). After being washed for five times with TBST for $10 \mathrm{~min}$, the membranes were incubated with the indicated secondary antibodies (horseradish peroxidase (HRP)-conjugated anti-mouse IgG (Santa Cruz, United States) and HRP-conjugated anti-rabbit IgG (Biosharp, China) (1:5,000 dilution)) for $1 \mathrm{~h}$ at room temperature. Then the protein bands were visualized with an ECL reagents (Millipore, United States) using Image Lab software (Bio-Rad Laboratories Inc., Hercules, CA, United States). GAPDH was viewed as an internal control. Finally, Image J (National Institute of Mental Health, United States) software was used to quantify the intensity of the protein bands.

\section{Dual-Luciferase Reporter Assay}

The indicated plasmids of COL1A1 promoter and the pRL-TK reporter plasmids were cotransfected into LX-2 cells according to the manufacturer's instructions of FuGENE (Promega, United States). After transfection for $18 \mathrm{~h}, \mathrm{LX}-2$ cells were treated with $\mathrm{rSjP} 40$ or not. The cells were then harvested after $48 \mathrm{~h}$ of stimulation. Dual-luciferase reporter assay was performed and the firefly and renilla luciferase activities were detected.

\section{Ets-1 Interference Experiment}

LX-2 cells were seeded in a twelve-well plate at a density of $5 \times 10^{4}$ cells per well. When cultured to $70-90 \%$ confluency, $4 \mu \mathrm{L}$ of lipofectamine 2000 reagent (Invitrogen, United States) was diluted with $100 \mu \mathrm{L}$ of DMEM and incubated at room temperature for $5 \mathrm{~min}$. Next, $2 \mu \mathrm{L}$ of siRNA of Ets-1 or negative control siRNA were combined with lipofectamine $2000 /$ medium mixture and allowed to complex by incubation for $20 \mathrm{~min}$ at room temperature. The mixed solution was added to $1 \mathrm{ml}$ of cell in the twelve -well plate. After transfection for 4-5 h, the culture medium was replaced with the fresh medium and the cells were incubated in the presence or absence of $20 \mu \mathrm{g} / \mathrm{ml} \mathrm{rSjP} 40$ for another $48 \mathrm{~h}$. 


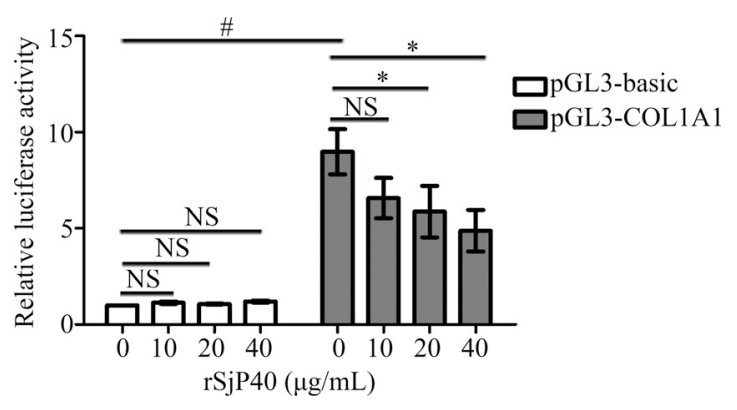

FIGURE 1 | rSjP40 inhibited COL1A1 promoter activity in LX-2 cells. The activity of COL1A1 promoter in LX-2 cells transfected with pGL3-COL1A1 was obviously enhanced than the luciferase activity in LX-2 cells transfected with pGL3-basic. $p<0.05$, compared to pGL3-basic+ rSjP40-group. rSjP40 could inhibit the activity of COL1A1 promoter in LX-2 cells transfected with pGL3-COL1A1. However, rSjP40 could not affect the luciferase activity in LX-2 cells transfected with pGL3-basic. $p<0.05$, compared to pGL3COL1A1 + rSjP40-group. NSP>0.05, compared to pGL3-COL1A1+ rSjP40group or pGL3-basic + rSjP40- group. NS, no significant.

\section{Chromatin Immunoprecipitation (ChIP)}

ChIP assay was carried out by SimpleChip Kit (Cell Signaling Technology, United States). Immunoprecipitation was performed with anti-Ets-1 antibody at $4^{\circ} \mathrm{C}$ overnight and normal IgG provided in SimpleChip Kit was used as a negative control. Precipitated DNA was analyzed by PCR using the primers as: 5'-CAATGGAATCTTGGATGG-3' (sense); 5' - TGAGAAACT CTGTAGGGC-3' (antisense), which were designed based on the Ets-1-binding sites on the COL1A1 promoter.

\section{Statistical Analysis}

All experiments were analyzed by the Student's $t$ test. All values included in the figures represent mean $\pm \mathrm{SD}$. A $p$-value $<0.05$ was considered significant.

\section{RESULTS}

\section{rSjP40 Down-Regulated the Activity of COL1A1 Promoter in LX-2 Cells}

In our previous studies, we have confirmed that $\mathrm{rSjP} 40$ could inhibit COL1A1 expression in LX-2 cells (Sun et al., 2015; Chen et al., 2016b). COL1A1 promoter is located upstream of transcription initiation and plays a key role in regulating COL1A1 transcription. In this study, we further investigated the effect of $\mathrm{rSjP} 40$ on the transcriptional activity of COL1A1 expression. We constructed the COL1A1 promoter plasmid pGL3-COL1A1 (-1,722/+21). The fluorescence reporter plasmid containing COL1A1 promoter was transfected into LX-2 and detected by dual luciferase reporter assay. Dual luciferase reporter gene assay showed that significantly higher activity was observed in pGL3-COL1A1 than pGL3-basic $(p<$ 0.05 , Figure 1). In addition, $\mathrm{rSjP} 40$ inhibited COL1A1 promoter activity in a concentration-dependent manner (Figure 1). Hence, we consider that $\mathrm{rSj} 40$ inhibits COL1Alexpression in LX-2 cells by inhibiting the activity of COL1A1 promoter.

\section{rSjP40 Down-Regulated the Promoter activity of COL1A1 at the Core Region of $-1,722 /-1,592$}

Further, we attempted to seek the core region at which $\mathrm{rSjP} 40$ could inhibit the promoter activity of COL1A1. We used bioinformatics to analyze COL1A1 promoter sequences and predict possible binding sites for common transcription factors. Truncated mutation of transcription factor binding site was carried out and five luciferase reporter gene plasmids were constructed for experimental study (Chen et al., 2019a): pGL3COL1A1a $(-1,592 /+21)$, pGL3-COL1A1b $(-1,167 /+21)$, pGL3COL1A1c $(-443 /+21)$, pGL3-COL1A1d $(-239 /+21)$, pGL3COL1Ale $(-215 /+21)$ (Figure 2A). Then the plasmids containing the truncated sequences of COL1A1 promoter (Chen et al., 2019a) were transfected into LX-2 cells, respectively. We found that $\mathrm{rSjP} 40$ could only inhibit the promoter activity in cells transfected with pGL3-COL1A1 (Figure 2B). However, rSjP40 could not affect the promoter activities in cells transfected with these truncated plasmids (Figure 2B). Compared the sequences of pGL3-COL1A1 and pGL3-COL1A1a (Chen et al., 2019a), we confirmed that rSjP40 down-regulated the promoter activity of COL1A1 at the core region of $-1,722 /-1,592$.

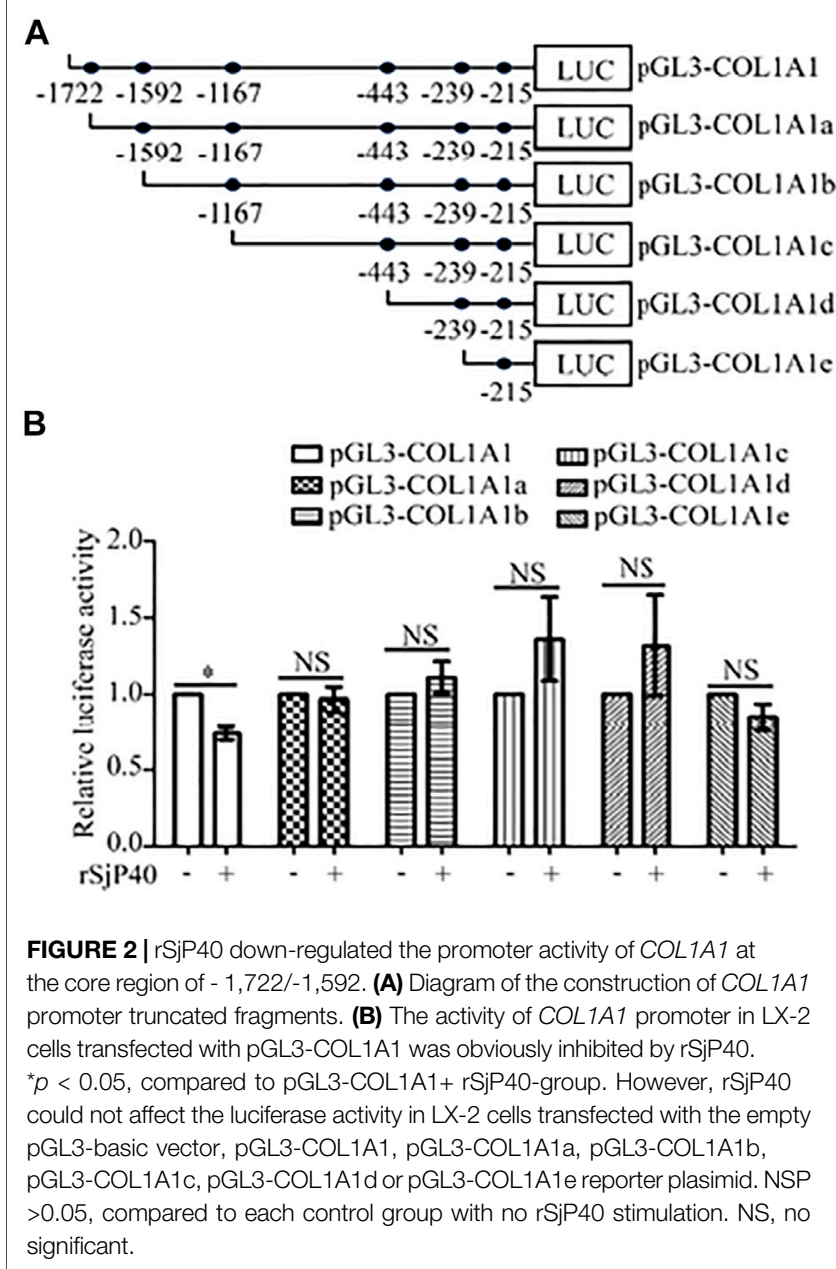




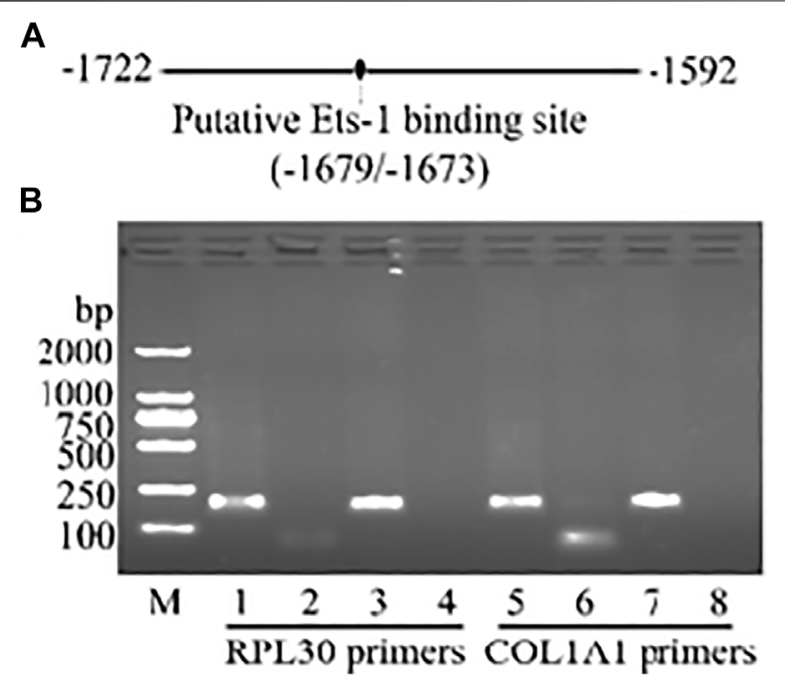

FIGURE 3 | Ets-1 may bind to COL1A1 promoter at the region of $-1,679 /-1,673$. (A) The binding site of transcriptional factor Ets-1 was predicted via JASPAR. (B) The combination of Ets-1 and collagen type I promoter was confirmed by ChIP method. Lanes 1-4, using RPL30 primers in the kit as the quality control experiment. Lanes 5-8, using COL1A1 promoter primers which were associated with Ets-1 binding site. Lane 1, antiHistone H3 group. Lane 5, anti-Ets-1 group. Lane 2 and lane 6, IgG group. Lane 3 and lane 7, Input. Lane 4 and lane 8, $\mathrm{dd}_{2} \mathrm{O}$ as the template of PCR. M, DL2000 DNA marker.

\section{Ets-1 May Bind to COL1A1 Promoter at the Region of $-1,679 /-1,673$}

To further explore whether transcription factors are involved in rSjP40 inhibiting COL1A1 promoter activity, we used JASPAR and PROMO database to predict the possible transcription factor binding sites in the main active region of COL1A1 promoter $-1,722 /-1,592$. The predicted transcription factor was Ets-1, which was located at $-1,679 /-1,673$ and shared similar loci in both databases (Figure 3A). Therefore, we hypothesized that rSjP40 might inhibit COL1A1 expression in LX-2 cells by regulating the transcription factor Ets-1. To verify this combination, ChIP analysis was performed and the results shown in Figure 3B confirmed that Ets-1 indeed combined to COL1A1 promoter at the region of $-1,679 /-1,673$. To perform the quality control experiment of ChIP kit, anti-Histone $\mathrm{H} 3$ antibody was used as the positive control and the template was then harvested to perform PCR using RPL30 primers provided in the ChIP kit.

\section{rSjP40 Inhibited the Expression of COL1A1 via Ets-1}

Previous studies have shown that Ets-1 is expressed in HSCs and regulates the transcription of ECM genes (Knittel et al., 1999). We further observed the expression of Ets-1 in $\mathrm{rSjP} 40$-treated LX-2 cells. The results of Western blot showed that $\mathrm{rSjP} 40$ could enhance the expression of Ets-1 in LX-2 cells (Figure 4A). To further confirm that $\mathrm{rSjP} 40$ inhibits the COL1A1 promoter by

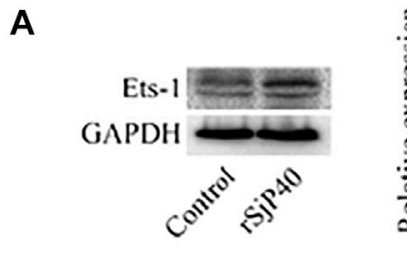

B
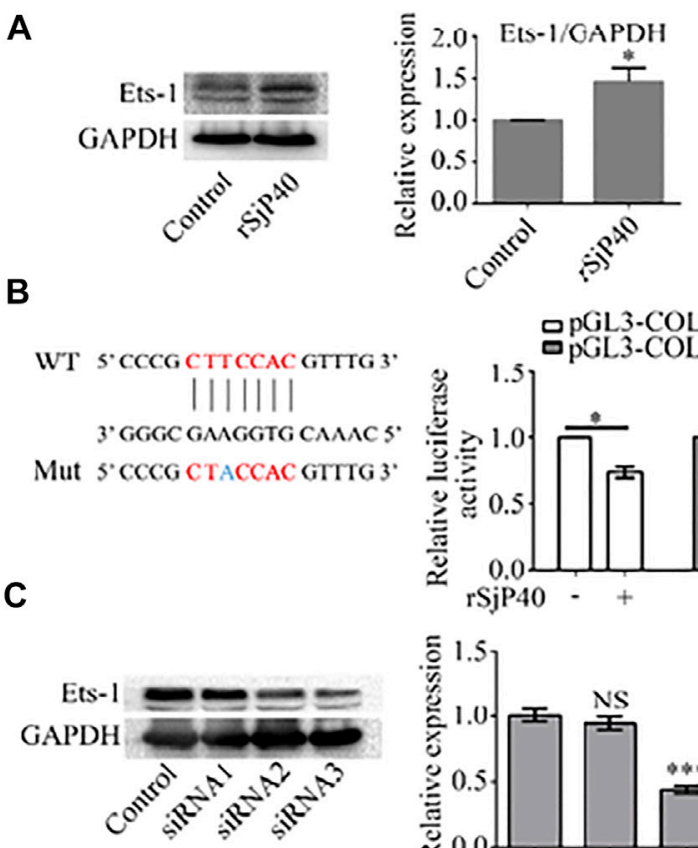

D
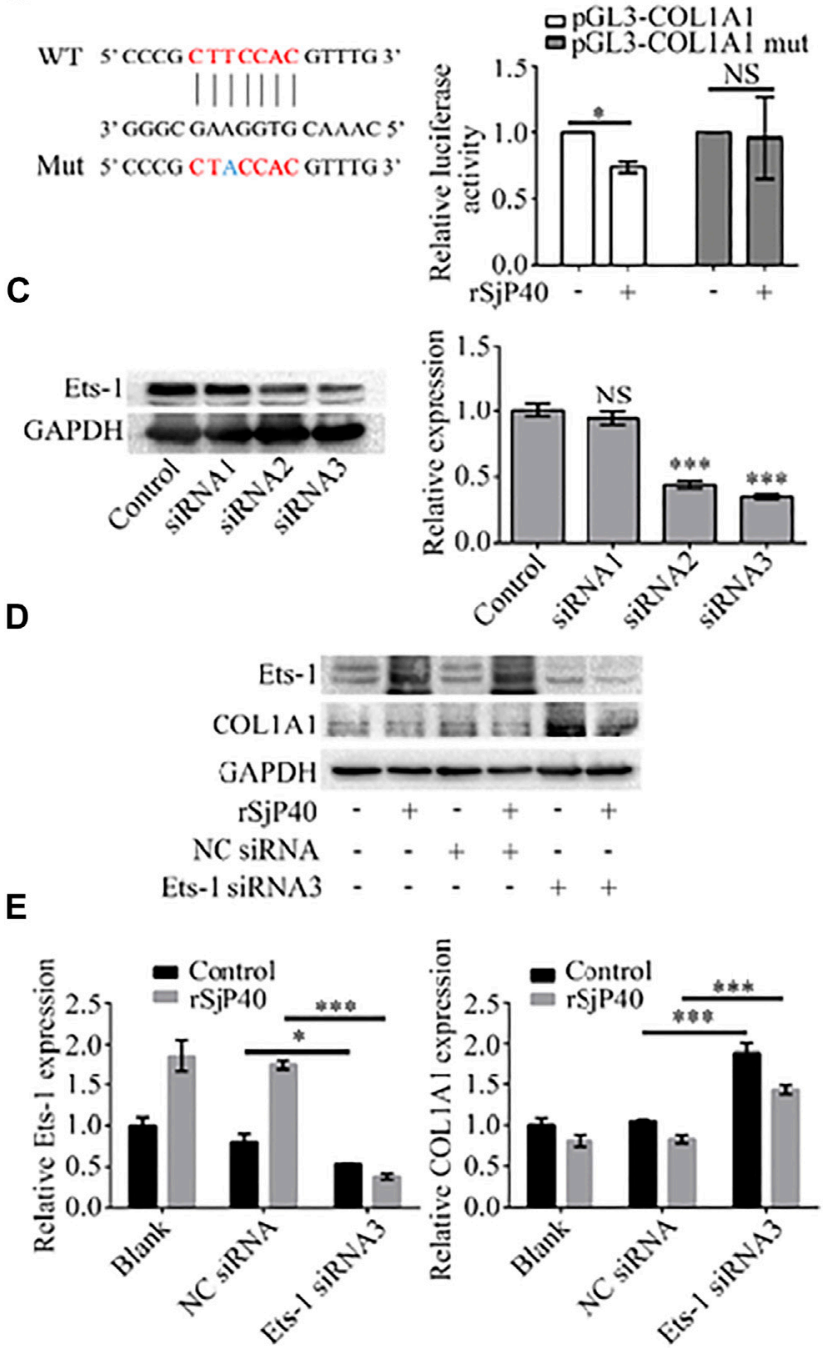

FIGURE 4 | rSjP40 inhibited the expression of COL1A1 via Ets-1. (A) rSjP40 could enhance the expression of Ets-1 protein in LX-2 cells. ${ }^{*} p<0.05$, compared to control group. (B) Diagram of SP1-binding site in the COL1A1 promoter region $-1,679 /-1,673$ and Ets-1 binding site mutant was showed (left). Red represents the ETS-1 binding region and blue represents the mutation site. rSjP40 could inhibit the luciferase activity of COL1A1 promoter (pGL3-COL1A1), but not inhibit the luciferase activity of the mutated COL1A1 promoter (pGL3-COL1A1 mut) (right). NSP >0.05, compared to control group with no rSjP40 stimulation. (C) siRNA NC or siRNA Ets-1 was transfected into LX-2 cells. The downregulated Ets-1 was confirmed by western blot $72 \mathrm{~h}$ post transfection. (D) Western blot results showed that a higher level of COL1A1 in siRNA Ets-1 cells than that in the siRNA NC group. (E) Quantification of western blot data for Ets-1 and COL1A1 in LX-2 cells. ${ }^{*} p<$ $0.05, p<0.001$ compared to siRNA NC group. WT, wild type; Mut, mutant; NS, no significant; NC: Negative control. 
regulating the transcription factor Ets-1, we mutated the base "T" at the ETS-1 binding site $-1,677$ on the COL1A1 promoter into "A" and labeled it as pGL3-COL1A1 mut. The effect of rSjP40 on COL1A1 promoter was detected by dual luciferin reporter gene. We found that $\mathrm{rSjP} 40$ could inhibit the luciferase activity of COL1A1 promoter (pGL3-COL1A1, Figure 4B). However, when the sequence of $-1,679 /-1,673$ was mutated, rSjP40 could not inhibit the luciferase activity of the mutated COL1A1 promoter (pGL3-COL1A1 mut, Figure 4B). These results suggested that Ets-1 played a negative regulatory role in the COL1A1 promoter. Then we further explored whether rSjP40 affects COL1A1 expression by regulating Ets-1. Successful knockdown of Ets-1 in LX-2 cells were confirmed by western blot (Figure 4C). And knockdown of Ets-1 reversed rSjP40-induced down-regulation of COL1A1 expression (Figures 4D,E). These results indicated that $\mathrm{rSjP} 40$ inhibited COL1A1 promoter activity and COL1A1 expression in LX-2 cells through ETS-1 dependent mechanism.

\section{DISCUSSION}

Liver fibrosis is a typical response to chronic liver disease and is characterized by large and excessive extracellular matrix in the liver. Currently, liver fibrosis is considered to be an evolutionarily conserved wound healing response to tissue injury, primarily driven by inflammatory and immune-mediated mechanisms (Pellicoro et al., 2014). Liver fibrosis is a dynamic and bidirectional process in which the interaction of multiple molecules, pathways and systems determines the self-limiting and dynamic equilibrium of fibrosis (Pellicoro et al., 2014; Sun and Kisseleva, 2015). HSCs are the main effector cells in the process of liver fibrosis. After chronic liver injury, they are transformed into myofibroblast-like cells, which unbalance the formation and degradation of extracellular matrix proteins and release pro-inflammatory and pro-fibrotic factors (Tang, 2015). Activated HSCs are the main source of collagen products, which increase the expression of integrin $\alpha 5 \beta 1$, and increases collagen synthesis through interactions between $\alpha 5 \beta 1$ and the extracellular matrix (Senoo et al., 2010). COL1A1 plays a dominant role in fibrotic scarring and protects liver cells from various harmful stimuli in the early stages of liver injury (Bourbonnais et al., 2012). However, when sustained damage leads to altered tissue function, excessive scarring can be caused and fibrosis can develop in an adverse direction.

Schistosomiasis is mainly caused by Schistosoma japonicum and Schistosoma mansoni, which are inflammatory diseases that cause fibrosis and portal hypertension (Chen et al., 2019b). These pathological changes are caused by the secretion of SEA by cercaria in the deposited eggs, which destroys the normal tissues of the host through chronic granulomatous inflammation mediated by T lymphocytes (Pearce and MacDonald, 2002; Ren et al., 2016). However, in addition to causing granuloma through immune mechanism, SEA also has a reverse regulation effect on liver fibrosis. Previous studies in our laboratory confirmed that SEA can induce the senescence and apoptosis of HSCs and inhibit the activation of LX-2 cells to inhibit liver fibrosis (Duan et al., 2014; Chen et al., 2016a). The main component of SEA is SjP40, which is the homologous protein of SmP40, the main egg component of Schistosoma mansoni, and they have high homology in amino acid sequence. SjP40 is often deposited in tissues in clusters to form larger granulomas. It has been reported that $\mathrm{SjP} 40$ and its antibody can be detected from the host as early as 21 days after the infection of Schistosoma japonicum, and is considered as a potential candidate antigen for the early diagnosis of schistosomiasis (Zhou et al., 2010). Then whether SjP40 has an effect on collagen prompted us to further explore the role of $\mathrm{SjP} 40$ and prepare recombinant Schistosoma japonicum egg protein rSjP40. Our results indicate that $\mathrm{rSjP} 40$ can inhibit the expression of COL1A1 in LX-2 cells, which is consistent with our previous results, demonstrating that $\mathrm{rSjP} 40$ can inhibit liver fibrosis by inhibiting the synthesis of COL1A1 in HSCs (Chen et al., 2016b). In this study, we focused on exploring the correlation between $\mathrm{rSjP} 40$ and the COL1A1 promoter. The results of this study proved that $\mathrm{rSjP} 40$ can inhibit the activity of $C O L 1 A 1$ promoter. At the same time, it was confirmed for the first time that the core region of $\mathrm{rSjP} 40$ acting on COL1A1 promoter was located at -1,722/-1,592.

As one of the most widely studied transcription factors in the Ets family, Ets-1 is involved in cell proliferation and apoptosis, angiogenesis, and tumor progression (Raouf and Seth, 2000). Studies have shown that Ets-1 is a known effector of mitogenactivated protein kinase (MAPK) pathway and a downstream target of extracellular regulated protein kinase (ERK), which effectively regulates the expression of genes related to endothelial cell growth and migration (Ito et al., 2004). Ets-1 has been shown to be expressed in HSCs and can regulate the transcription of ECM genes (Knittel et al., 1999). However, the role of Ets-1 in promoting or inhibiting liver fibrosis remains controversial. M Mizui et al. (Mizui et al., 2006) showed that overexpression of Ets- 1 could inhibit the production of COL1A1 in mesangial cells induced by TGF- $\beta$, so Ets- 1 is considered as an effective inhibitor of collagen synthesis. In contrast, Dechen Liu et al. (Liu et al., 2019) showed that the knockdown of Ets-1 reduced hepatocyte apoptosis and slowed down the progression of non-alcoholic steatohepatitis, protecting the liver from injury, inflammation and fibrosis. In our study, we found that $\mathrm{rSjP} 40$ could promote the expression of Ets-1 in human LX-2 cells. Studies have shown that IL-18 down-regulates collagen expression by activating Ets-1 through ERK pathway and directly inhibiting the activity of collagen promoter in human skin fibroblasts (Kim et al., 2010). Therefore, we hypothesized whether $\mathrm{rSjP} 40$ affects $C O L 1 A 1$ promoter activity by regulating Ets-1. CHIP experiments showed that Ets- 1 could directly bind to the COL1A1 promoter at the site-1,679/-1,673. Then we mutated the base "T" at $-1,677$ of the Ets-1 binding site on the COL1A1 promoter into "A", and the activity of the COL1A1 promoter significantly increased, indicating that Ets-1 has a negative regulation effect on the COL1A1 promoter in human LX-2 cells. In addition, $\mathrm{rSjP} 40$ could not inhibit the activity of the mutated $C O L 1 A 1$ promoter at the Ets-1 binding site, further confirming the role of $\mathrm{rSjP} 40$ by affecting Ets-1. Finally, 
knockdown of Ets-1 in LX-2 cells reversed rSjP40-induced downregulation of COL1A1 expression.

In conclusion, this study indicates that $\mathrm{rSjP} 40$ inhibits the activity of COL1A1 promoter by increasing the expression of transcription factor Ets-1, down-regulating the expression of COL1A1, thereby inhibiting the activation of HSCs and inhibiting liver fibrosis. The results of this study will provide a new experimental basis for the prevention and treatment of liver fibrosis.

\section{DATA AVAILABILITY STATEMENT}

The original contributions presented in the study are included in the article/Supplementary Material, further inquiries can be directed to the corresponding authors.

\section{AUTHOR CONTRIBUTIONS}

YD and LD: conception and design, financial support, and final approval of the manuscript. JL, JZ, and BZ: manuscript writing,

\section{REFERENCES}

Bourbonnais, É., Raymond, V. A., Éthier, C., Nguyen, B. N., El-Leil, M. S., Meloche, S., et al. (2012). Liver Fibrosis Protects Mice from Acute Hepatocellular Injury. Gastroenterology 142, 130-139. doi:10.1053/j.gastro.2011.09.033

Chatterjee, S., Vrolix, G., Depoortere, I., Peeters, T., and Van Marck, E. (2005). The Therapeutic Effect of the Neuropeptide Hormone Somatostatin on Schistosoma Mansoni Caused Liver Fibrosis. BMC Infect. Dis. 5, 45. doi:10.1186/1471-23345-45

Chen, J., Pan, J., Wang, J., Song, K., Zhu, D., Huang, C., et al. (2016a). Soluble Egg Antigens of Schistosoma Japonicum Induce Senescence in Activated Hepatic Stellate Cells by Activation of the STAT3/p53/p21 Pathway. Sci. Rep. 6, 30957. doi:10.1038/srep30957

Chen, J., Xu, T., Zhu, D., Wang, J., Huang, C., Lyu, L., et al. (2016b). Egg Antigen P40 of Schistosoma Japonicum Promotes Senescence in Activated Hepatic Stellate Cells by Activation of the STAT3/p53/p21 Pathway. Cell Death Dis 7, e2315. doi:10.1038/cddis.2016.228

Chen, L., Ji, Z., Duan, L., Zhu, D., Chen, J., Sun, X., et al. (2019a). rSJYB1 Inhibits Collagen Type I Protein Expression in Hepatic Stellate Cells via Downregulating Activity of Collagen al (I) Promoter. J. Cel Mol Med 23, 3676-3682. doi:10.1111/jcmm.14271

Chen, T. T. W., Cheng, P. C., Chang, K. C., Cao, J. P., Feng, J. L., Chen, C. C., et al. (2019b). Activation of the NLRP3 and AIM2 Inflammasomes in a Mouse Model ofSchistosoma Mansoniinfection. J. Helminthol. 94, e72. doi:10.1017/ S0022149X19000622

Duan, Y., Gu, X., Zhu, D., Sun, W., Chen, J., Feng, J., et al. (2014). Schistosoma Japonicum Soluble Egg Antigens Induce Apoptosis and Inhibit Activation of Hepatic Stellate Cells: a Possible Molecular Mechanism. Int. J. Parasitol. 44, 217-224. doi:10.1016/j.ijpara.2013.11.003

Eckes, B., Zigrino, P., Kessler, D., Holtkötter, O., Shephard, P., Mauch, C., et al. (2000). Fibroblast-matrix Interactions in Wound Healing and Fibrosis. Matrix Biol. 19, 325-332. doi:10.1016/s0945-053x(00)00077-9

Fabregat, I., and Caballero-Díaz, D. (2018). Transforming Growth Factor$\beta$-Induced Cell Plasticity in Liver Fibrosis and Hepatocarcinogenesis. Front. Oncol. 8, 357. doi:10.3389/fonc.2018.00357

Ito, H., Duxbury, M., Benoit, E., Clancy, T. E., Zinner, M. J., Ashley, S. W., et al. (2004). Prostaglandin E2Enhances Pancreatic Cancer Invasiveness through an Ets-1-dependent Induction of Matrix Metalloproteinase-2. Cancer Res. 64, 7439-7446. doi:10.1158/0008-5472.CAN-04-1177 assembly of data, and data analysis and interpretation. LC and GC: assembly of data. DZ and JC: data analysis and interpretation. All authors contributed to the article and approved the submitted version.

\section{FUNDING}

This work was supported by National Natural Science Foundation of China (Grant Numbers 81871677, 82172295, 81471975) and the Natural Science Foundation of Nantong City (JC2020021). The funders had no role in study design, data collection and analysis, decision to publish, or preparation of the manuscript.

\section{ACKNOWLEDGMENTS}

LX-2 cells were kindly present from Zhaolian Bian (Nantong Institute of Liver disease, Nantong Third People's Hospital, Nantong, China).

Kim, H. J., Song, S. B., Choi, J. M., Kim, K. M., Cho, B. K., Cho, D. H., et al. (2010) IL-18 Downregulates Collagen Production in Human Dermal Fibroblasts via the ERK Pathway. J. Invest. Dermatol. 130, 706-715. doi:10.1038/jid.2009.302 Knittel, T., Kobold, D., Dudas, J., Saile, B., and Ramadori, G. (1999). Role of the Ets1 Transcription Factor during Activation of Rat Hepatic Stellate Cells in Culture. Am. J. Pathol. 155, 1841-1848. doi:10.1016/S0002-9440(10)65502-2

Liu, D., Wang, K., Li, K., Xu, R., Chang, X., Zhu, Y., et al. (2019). Ets-1 Deficiency Alleviates Nonalcoholic Steatohepatitis via Weakening TGF-B1 SignalingMediated Hepatocyte Apoptosis. Cel Death Dis 10, 458. doi:10.1038/s41419019-1672-4

Liu, T., Wang, P., Cong, M., Zhang, D., Liu, L., Li, H., et al. (2016). Matrix Metalloproteinase-1 Induction by Diethyldithiocarbamate Is Regulated via Akt and ERK/miR222/ETS-1 Pathways in Hepatic Stellate Cells. Biosci. Rep. 36, e00371. doi:10.1042/BSR20160111

Mizui, M., Isaka, Y., Takabatake, Y., Sato, Y., Kawachi, H., Shimizu, F., et al. (2006). Transcription Factor Ets-1 Is Essential for Mesangial Matrix Remodeling. Kidney Int. 70, 298-305. doi:10.1038/sj.ki.5001541

Ozaki, I., Mizuta, T., Zhao, G., Zhang, H., Yoshimura, T., Kawazoe, S., et al. (2003). Induction of Multiple Matrix Metalloproteinase Genes in Human Hepatocellular Carcinoma by Hepatocyte Growth Factor via a Transcription Factor Ets-1. Hepatol. Res. 27, 289-301. doi:10.1016/s1386-6346(03)00268-7

Ozaki, I., Zhao, G., Mizuta, T., Ogawa, Y., Hara, T., Kajihara, S., et al. (2002). Hepatocyte Growth Factor Induces Collagenase (Matrix Metalloproteinase-1) via the Transcription Factor Ets-1 in Human Hepatic Stellate Cell Line. J. Hepatol. 36, 169-178. doi:10.1016/s0168-8278(01)00245-8

Pearce, E. J., and MacDonald, A. S. (2002). The Immunobiology of Schistosomiasis. Nat. Rev. Immunol. 2, 499-511. doi:10.1038/nri843

Pellicoro, A., Ramachandran, P., Iredale, J. P., and Fallowfield, J. A. (2014). Liver Fibrosis and Repair: Immune Regulation of Wound Healing in a Solid Organ. Nat. Rev. Immunol. 14, 181-194. doi:10.1038/nri3623

Raouf, A., and Seth, A. (2000). Ets Transcription Factors and Targets in Osteogenesis. Oncogene 19, 6455-6463. doi:10.1038/sj.onc.1204037

Ren, J., Hu, L., Yang, J., Yang, L., Gao, F., Lu, P., et al. (2016). Novel T-Cell Epitopes onSchistosoma japonicumSjP40 Protein and Their Preventive Effect on Allergic Asthma in Mice. Eur. J. Immunol. 46, 1203-1213. doi:10.1002/eji.201545775

Senoo, H., Yoshikawa, K., Morii, M., Miura, M., Imai, K., and Mezaki, Y. (2010). Hepatic Stellate Cell (Vitamin A-Storing Cell) and its Relative - Past, Present and Future. Cell. Biol. Int. 34, 1247-1272. doi:10.1042/CBI20100321

Sun, M., and Kisseleva, T. (2015). Reversibility of Liver Fibrosis. Clin. Res. Hepatol. Gastroenterol. 39 (Suppl. 1), S60-S63. doi:10.1016/j.clinre.2015.06.015 
Sun, X., Zhang, L., Wang, J., Chen, J., Zhu, D., Shen, P., et al. (2015). Schistosoma Japonicum Protein SjP40 Inhibits TGF-B1-Induced Activation of Hepatic Stellate Cells. Parasitol. Res. 114, 4251-4257. doi:10.1007/s00436-015-4663-0

Tang, Y. (2015). Curcumin Targets Multiple Pathways to Halt Hepatic Stellate Cell Activation: Updated Mechanisms In Vitro and In Vivo. Dig. Dis. Sci. 60, 1554-1564. doi:10.1007/s10620-014-3487-6

Udomsinprasert, W., and Jittikoon, J. (2019). Vitamin D and Liver Fibrosis: Molecular Mechanisms and Clinical Studies. Biomed. Pharmacother. 109, 1351-1360. doi:10.1016/j.biopha.2018.10.140

Yang, Y., Kim, H. J., Woo, K.-J., Cho, D., and Bang, S. I. (2017). Lipo-PGE1 Suppresses Collagen Production in Human Dermal Fibroblasts via the ERK/ Ets-1 Signaling Pathway. PLoS One 12, e0179614. doi:10.1371/ journal.pone.0179614

Yin, C., Evason, K. J., Asahina, K., and Stainier, D. Y. R. (2013). Hepatic Stellate Cells in Liver Development, Regeneration, and Cancer. J. Clin. Invest. 123, 1902-1910. doi:10.1172/JCI66369

Zhou, X.-H., Wu, J.-Y., Huang, X.-Q., Kunnon, S. P., Zhu, X.-Q., and Chen, X.-G. (2010). Identification and Characterization of Schistosoma Japonicum Sjp40, a Potential Antigen Candidate for the Early Diagnosis of Schistosomiasis. Diagn. Microbiol. Infect. Dis. 67, 337-345. doi:10.1016/j.diagmicrobio.2010.03.003
Zhu, D., Hu, B., Zhou, Y., Sun, X., Chen, J., Chen, L., et al. (2018). microRNA-146a Is Involved in $\mathrm{rSjP} 40$-inhibited Activation of LX-2 Cells by Targeting Smad4 Expression. J. Cel Biochem 119, 9249-9253. doi:10.1002/jcb.27193

Conflict of Interest: The authors declare that the research was conducted in the absence of any commercial or financial relationships that could be construed as a potential conflict of interest.

Publisher's Note: All claims expressed in this article are solely those of the authors and do not necessarily represent those of their affiliated organizations, or those of the publisher, the editors and the reviewers. Any product that may be evaluated in this article, orclaim that may be made by its manufacturer, is not guaranteed or endorsed by the publisher.

Copyright (C) 2021 Li, Zhang, Zhang, Chen, Chen, Zhu, Chen, Duan and Duan. This is an open-access article distributed under the terms of the Creative Commons Attribution License (CC BY). The use, distribution or reproduction in other forums is permitted, provided the original author(s) and the copyright owner(s) are credited and that the original publication in this journal is cited, in accordance with accepted academic practice. No use, distribution or reproduction is permitted which does not comply with these terms. 